\title{
Communicable Diseases Report, NSW, January and February 2011
}

\section{Communicable Diseases Branch NSW Department of Health}

For updated information, including data and facts on specific diseases, visit www.health.nsw.gov.au and click on Public Health and then Infectious Diseases. The communicable diseases site is available at: http://www.health.nsw.gov.au/ publichealth/infectious/index.asp.

Figure 1 and Tables 1 and 2 show reports of communicable diseases received through to the end of January and February 2011 in New South Wales (NSW).

\section{Enteric infections \\ Outbreaks of foodborne disease}

Eighteen outbreaks of suspected foodborne disease were investigated in January and February 2011. These outbreaks were identified through surveillance of laboratory notifications, or complaints to the NSW Food Authority or the local public health unit (PHU). In seven of these outbreaks the causative organism was identified as Salmonella enterica serovar Typhimurium and in another, $S$. Singapore.

Four of the $S$. Typhimurium outbreaks were identified through laboratory notifications. Interviews with the affected people found likely food items and sources in each of these outbreaks. For one of these outbreaks, illness was found to be associated with a dessert containing partially cooked eggs served in a restaurant over 3 nights. Another outbreak, with 82 confirmed cases, was associated with Vietnamese pork rolls containing a raw egg product from a Vietnamese bakery. A third outbreak was found to be associated with cake, possibly cross-contaminated with a piping bag, from a bakery. The fourth outbreak was found to be associated with fried ice-cream made with raw egg from a Chinese restaurant.

The remaining three $S$. Typhimurium outbreaks were identified by the NSW Food Authority or the local PHU following reports of gastrointestinal illness. The first report was from several separate groups who were ill after eating a chicken caesar salad with a dressing made from raw eggs at a café. The second report was from a boarding school where 26 out of 260 students developed illness. A cohort study found the only food associated with illness to be apple turnover. The association was weak (odds ratio $=4.6,95 \%$ CI 1.4-15.4) and no high risk ingredients were included in this apple turnover. The third report was from a family who ate battered lamb at a restaurant. One person in the family was reported to be unwell and the PHU could not rule out other possible exposures.

The $S$. Singapore outbreak occurred on a cruise and affected two party groups. A cohort study revealed roast chicken to be the likely pathogen source (odds ratio $=26.4$, 95\% CI 2.9-244.4). The cooked chicken was purchased from a supermarket, where it was potentially contaminated by raw chicken.

Salmonella infections can occur after eating undercooked food made from eggs, meat or poultry. ${ }^{1}$ Sometimes it can be spread by contact with a person with the infection, or if an infected person has prepared food for others. Thorough cooking of food kills Salmonella bacteria. The best way to avoid contracting a Salmonella infection is to avoid raw or undercooked meat, poultry or eggs. Poultry and meatsuch as hamburgers, sausages and rolled roasts - should not be eaten if pink in the middle.

An outbreak of Campylobacter enteritis in guests attending a birthday party function at a restaurant was epidemiologically linked to poultry liver pate. Eleven guests developed symptoms consistent with Campylobacter enteritis and stool culture confirmed this for two. The dish was prepared by briefly flash frying intact chicken livers then mixing these with other ingredients but not cooking further. Poultry liver tissue is frequently contaminated with Campylobacter bacteria and deliberate undercooking has been implicated in previous outbreaks.

\section{Outbreaks of gastroenteritis in institutional settings}

During January and February, 61 outbreaks of gastroenteritis in institutions were reported, affecting 614 people. Thirty outbreaks occurred in child care centres, 26 in aged care facilities, four in hospitals, and one in a residential care facility. The majority of outbreaks (93\%) appeared to have been caused by person-to-person spread of a viral illness. In 28 outbreaks (46\%) one or more stool specimens were collected. In six of these outbreaks (21\%) norovirus 
was detected, in two outbreaks (7\%) Giardia bacteria was detected and in one outbreak (4\%) stool specimens tested positive for both rotavirus and Clostridium difficile. Stool specimens were not available for laboratory testing for the remaining 33 outbreaks.

Sixty-one outbreaks is about double the average number of outbreaks for January and February combined for the last 5 years (which was 32). Viral gastroenteritis tends to decline in warmer months. The increase in reports of gastroenteritis in this period was largely attributable to an increased number of reports from child care centres at $240 \%$ the previous 5 -year average for the same time period. In contrast, the increase in aged care facility outbreaks was only $170 \%$ the previous 5-year average and in hospitals, a 7\% decrease. The apparent increase in outbreaks from child care may be due to improved reporting from centres. Public health units are encouraging parents to submit stool specimens from their children for laboratory testing to determine the cause of the infections.

\section{Respiratory and other infections Influenza}

During January and February influenza activity was low in NSW, as measured by the number of patients who presented to 56 of the state's largest emergency departments with influenza-like-illness. There were 110 emergency department presentations of patients with influenza-like illness (1.0 per 1000 presentations) for January and 76 presentations ( 0.5 per 1000 presentations $)$ for February.

The number of patients who tested positive for influenza at diagnostic laboratories was slightly above the usual level for this time of year. There were 58 cases of influenza confirmed by direct immunofluorescence or polymerase chain reaction testing in January and 43 cases in February. Of these, 68\% were identified as the pandemic (H1N1) 2009 virus.

For a more detailed report on respiratory activity in NSW see: http://www.health.nsw.gov.au/PublicHealth/ Infectious/influenza_reports.asp

\section{Vaccine-preventable diseases Measles}

Twenty cases of measles were reported in January and February (one in January and 19 in February), compared to five for the same period in 2010. Five cases in this period were associated with overseas travel (two from the Philippines, two from Italy and one from France).

Fourteen linked cases were identified in Western Sydney. The first case notified (whose source remains unknown) attended a social gathering while infectious. From this case, two further cases were reported in the community from child contacts who were unvaccinated or who had received only one dose of measles-containing vaccine. Five subsequent cases were reported from a local high school and six cases from the local community with no identified links to known cases of measles.

Measles virus is especially infectious and is easily spread among unvaccinated or partially vaccinated people. The virus is spread through the air by someone who is unwell with the disease. Symptoms include fever, sore eyes and a cough followed a few days later by a rash. ${ }^{1}$

Many people who were born during or since 1966 may not be immune to measles because they have neither been infected with measles nor received two doses of a measlescontaining vaccine. People who are planning overseas travel should ensure they have received two doses of the free measles-mumps-rubella vaccine (at least 1 month apart) from their general practitioner (GP) or at a travel health clinic. Measles vaccine is recommended for infants at 12 months and at 4 years of age, and this provides longlasting immunity in $99 \%$ of recipients. ${ }^{2}$

\section{Meningococcal disease}

Twelve cases of meningococcal disease were reported in NSW in January and February (seven in January and five in February). No links were identified between cases. The age of cases in this period ranged from 0 to 61 years and included five cases aged less than 5 years. No deaths were reported in this period. Five cases were caused by Neisseria meningitidis serogroup B, one case by N. meningitidis serogroup W135, and for six cases the serogroup was unable to be determined. In 2010, 73 cases of meningococcal disease were reported in NSW (including five deaths).

A free vaccine for serogroup $\mathrm{C}$ meningococcal disease is available for infants at 12 months of age. Consequently, serogroup $\mathrm{C}$ meningococcal disease is now mainly seen in adults and in unimmunised children. In NSW in 2010, 82\% of cases of meningococcal disease (where the serogroup was known) were caused by $N$. meningitidis serogroup B, for which there is no vaccine.

\section{Pertussis (whooping cough)}

During January and February, 2698 cases of pertussis were reported in NSW. This followed an increase in late 2010, with 1860 cases reported in November and 1590 cases in December. The number of reported cases was highest in children aged $0-4$ years and 5-9 years. In total, 9244 cases were reported in 2010 compared with 12577 in 2009.

A free vaccine is available for infants at 2, 4 and 6 months (although the first dose can be given as early as 6 weeks of 
age) with a booster dose at 4 years (which can be given as early as 3 years and 6 months of age). ${ }^{3}$ Immunisation reduces the risk of infection, however the vaccine does not provide lifelong protection, and re-infection can occur. ${ }^{2}$ Because pertussis immunity wanes over time, many older children and adults are susceptible to infection and can be the source of new infections in infants. ${ }^{4}$ For a limited time, NSW Health is providing free pertussis (dTpa) vaccine via GPs to all new parents, grandparents and any other adults who will regularly care for infants less than 12 months of age. Free vaccine boosters are also provided in high school as part of NSW Health's School-Based Vaccination Program.

\section{Sexually transmissible infections Gonorrhoea}

Notifications of gonorrhoea continued to decrease during January and February 2011, following a downward trend reported at the end of 2010. There were a total of 379 notifications of gonorrhoea reported (195 in January and 184 in February) in this period, compared to 445 (244 in January and 201 in February) in the same period in 2010 .

Gonorrhoea is a bacterial infection spread through vaginal, oral or anal sex without a condom. Infection in men can present as discharge from the penis, irritation or pain on urinating. Infections of the cervix, anus and throat usually cause no symptoms. ${ }^{1}$

\section{Syphilis}

Notifications of infectious syphilis continued to decrease during January and February 2011, following a significant decrease at the end of 2010. In total, 46 cases of infectious syphilis were reported in this period (28 in January and 18 in February). This is a decrease of approximately $40 \%$ compared to the same time period in 2010 (45 in January and 36 in February). The majority of notifications continue to occur in males aged between 20 and 50 years.

Syphilis is a highly infectious sexually transmitted disease that is spread through vaginal, anal or oral sex through skin-to-skin contact. Syphilis is highly contagious during the primary and secondary stages when the sore or rash is present. ${ }^{1}$ Those most at risk include men who have sex with men, people with HIV/AIDS, and people living in Aboriginal communities that are remote or have poor access to health care services.

\section{Lymphogranuloma venereum}

An outbreak of lymphogranuloma venereum (LGV) was identified in NSW in 2010 with a peak in notifications reported between May and August when 32 cases were reported. Since then, the number of notifications dropped significantly but increased during January and February 2011 with 10 cases reported.

LGV is a sexually transmitted infection. It is caused by a rare, severe strain of Chlamydia trachomatis which generally causes more severe symptoms than chlamydia. LGV is spread through unprotected vaginal, anal or oral sexual contact. ${ }^{1}$

\section{Mosquito-borne infections}

During January and February 2011, mosquitoes were found at very high levels in many parts of rural NSW, especially in southern parts of the state. Mosquitoes potentially carry a number of different viruses that can cause human disease.

The most common mosquito-borne infections of humans in NSW are Ross River virus and Barmah Forest virus infection, both of which can cause a prolonged illness with fever, rash, sore joints and fatigue. ${ }^{1}$

In this period 147 cases of Ross River virus infection were reported (64 in January and 83 February) compared to 184 cases for the same period in 2010. In total, 154 cases of Barmah Forest virus infection were reported in this period (96 in January and 58 February) compared to 49 cases for the same period in 2010 .

A number of sentinel chicken flocks are located around western NSW and Victoria to help monitor the risk of more serious mosquito-borne infections such as Murray Valley encephalitis (MVE) and Kunjin virus disease. MVE was detected in sentinel chickens in the Victorian towns of Mildura, Robinvale, Kerang and Barmah, and also in Tooleybuc in NSW. ${ }^{5}$ Although a number of people have been tested for these infections, no human cases have been confirmed at this stage.

In response to the infections in chickens, NSW Health commissioned a print and radio-based community awareness campaign to inform the public about how best to avoid mosquito bites to prevent mosquito-borne diseases. PHUs are maintaining regular contact with health facilities in western NSW to identify possible cases early and ensure that appropriate investigations are performed. PHUs have also disseminated information about MVE to local clinicians. The last human case of MVE detected in NSW was reported in 2008 .

\section{References}

1. Heymann DL, editor Control of Communicable Diseases Manual. 19th ed. Washington: American Public Health Association; 2008 
2. National Health and Medical Research Council. The Australian Immunisation Handbook. 9th ed. Canberra: Australian Government Department of Health and Ageing; 2008.

3. Australian Technical Group on Immunisation (ATAGI). 41st Meeting: 15-16 October 2009, summary of outcomes. Available from: http://immunise.health.gov.au/internet/ immunise/publishing.nsf/Content/E7E989916C4FCAD8 CA2576BF007D6B6E/\$File/ATAGI-41-bulletin.pdf (Cited 30 March 2010.)
4. Wendelboe AM, Njamkepo E, Bourillon A, Floret D, Gaudelus J, Gerber M. Transmission of Bordetella pertussis to young infants. Pediatr Infect Dis J 2007; 26: 293-9. doi:10.1097/01.inf.0000258699.64164.6d

5. NSW Department of Health media release. Available from: http://www.health.nsw.gov.au/news/2011/20110223_00.html (Cited 29 March 2011.) 
Figure 1. Reports of selected communicable diseases, NSW, January 2004 to February 2011, by month of onset. Preliminary data: case counts in recent months may increase because of reporting delays. Laboratory-confirmed cases only, except for measles, meningococcal disease and pertussis. BFV, Barmah Forest virus infection; RRV, Ross River virus infections; lab conf, laboratory confirmed; Men $G p C$ and $G p B$, meningococcal disease due to serogroup $C$ and serogroup $B$ infection; other/unk, other or unknown serogroups.

NB: Multiple series in graphs are stacked, except gastroenteritis outbreaks.

NB: Outbreaks are more likely to be reported by nursing homes $\&$ hospitals than by other institutions.

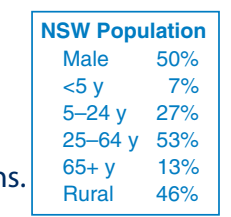

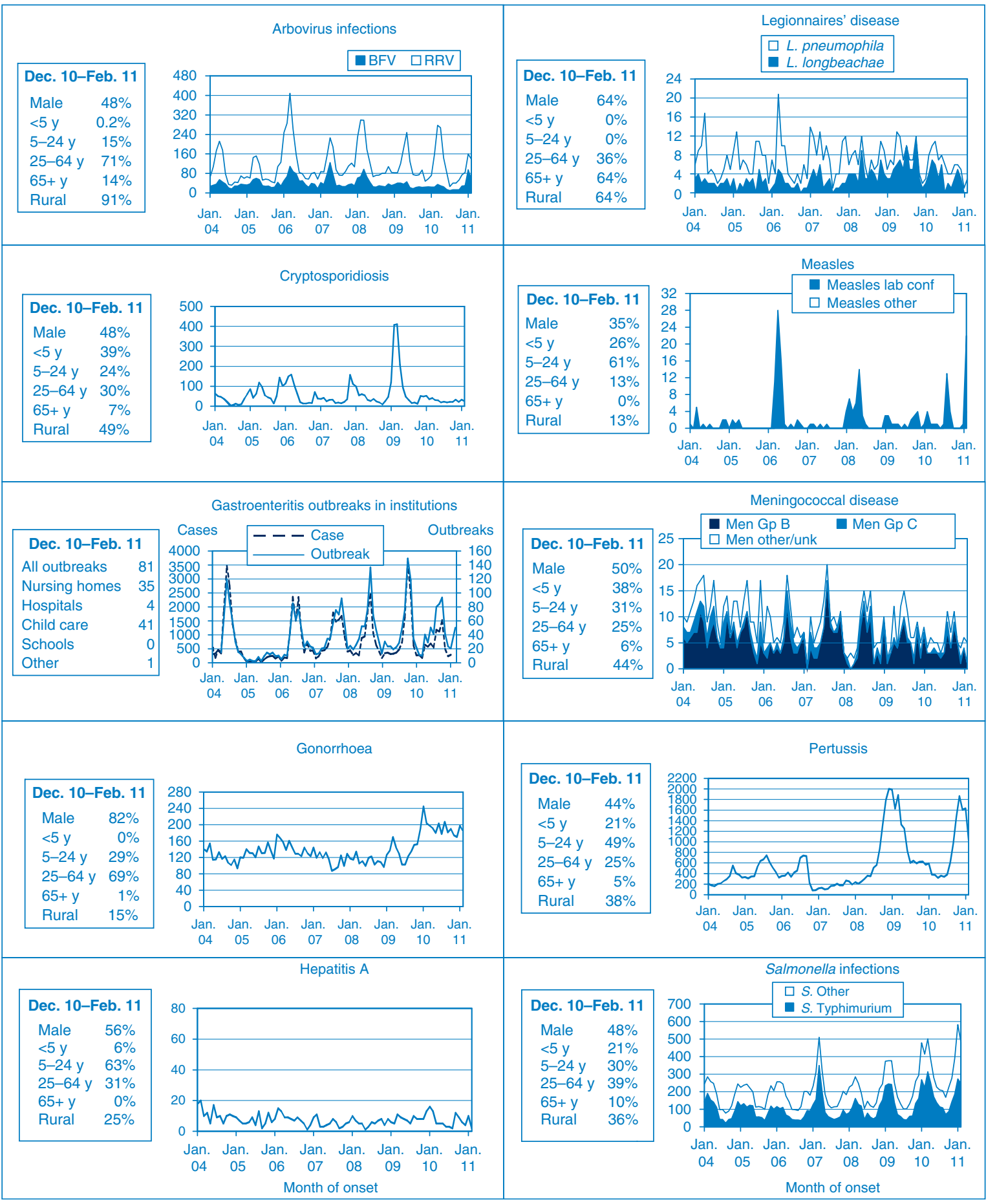




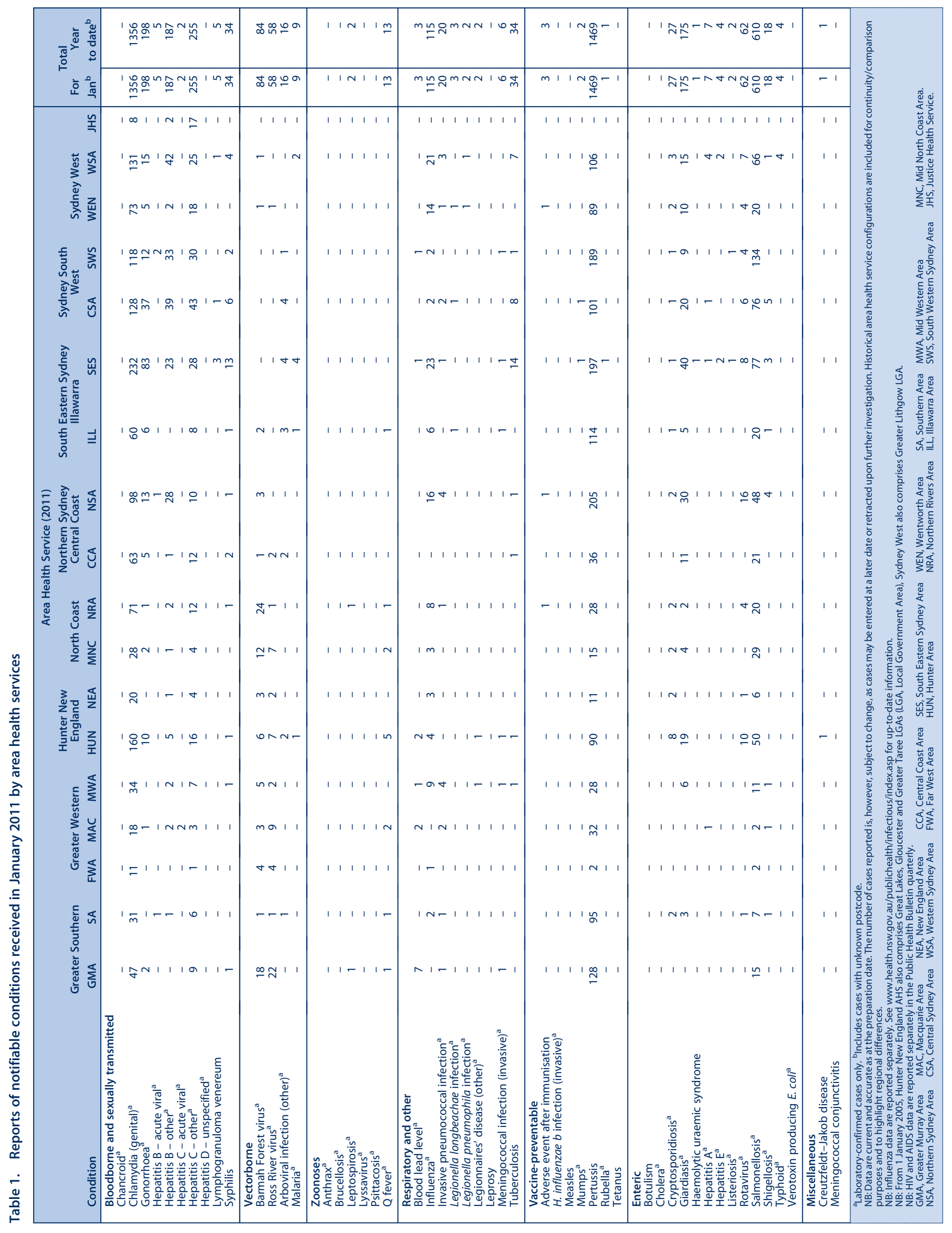




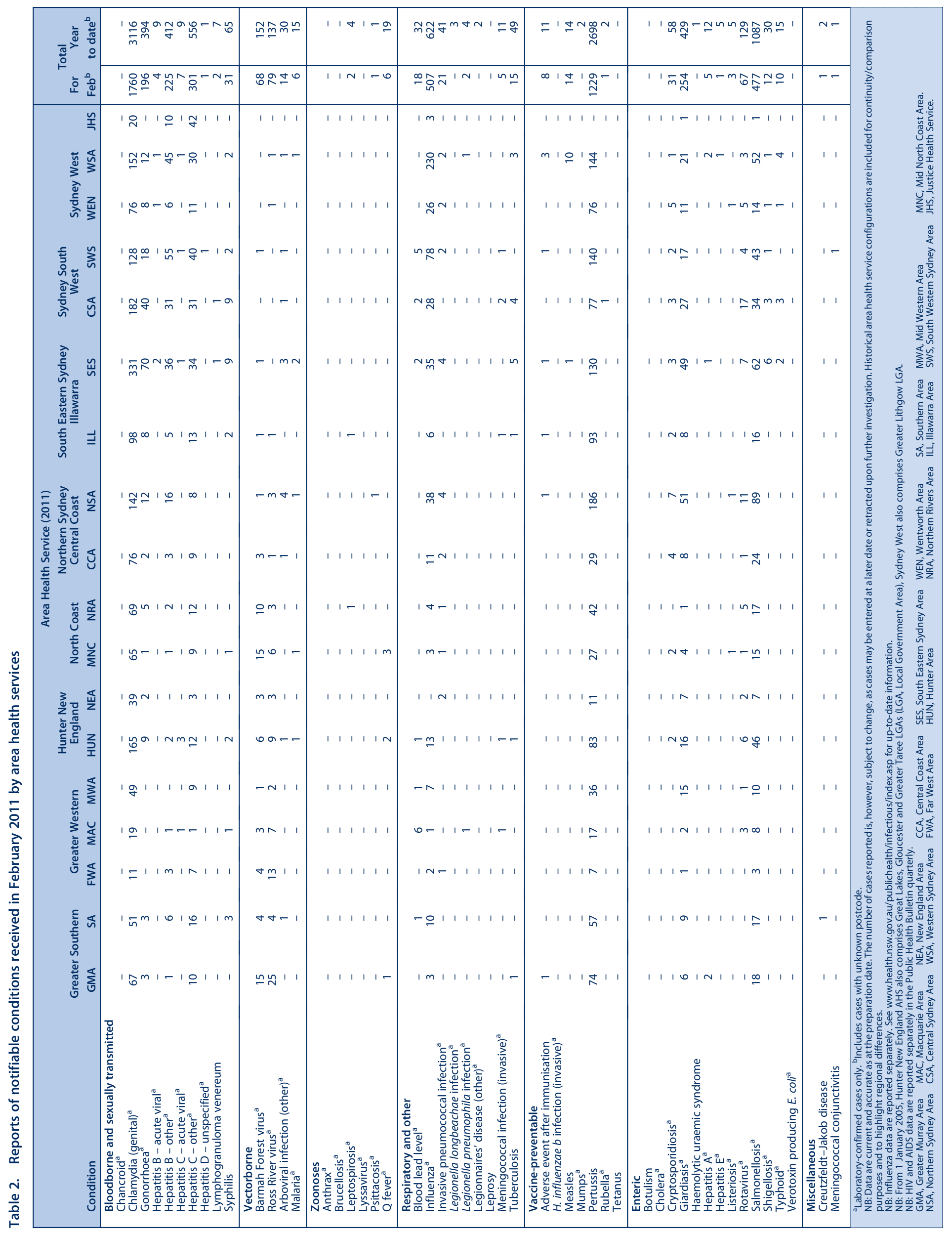

